\title{
THE CHEN-RUBIN CONJECTURE IN A CONTINUOUS SETTING*
}

\author{
CHRISTIAN BERG ${ }^{\dagger}$ AND HENRIK L. PEDERSEN ${ }^{\ddagger}$
}

\begin{abstract}
We study the median $m(x)$ in the gamma distribution with parameter $x$ and show that $0<m^{\prime}(x)<1$ for all $x>0$. This proves a generalization of a conjecture of Chen and Rubin from 1986: The sequence $m(n)-n$ decreases for $n \geq 1$. We also describe the asymptotic behaviour of $m$ and $m^{\prime}$ at zero and at infinity.
\end{abstract}

Key words. median, gamma function, gamma distribution

AMS subject classifications. primary 60E05; secondary 41A60, 33B15

1. Introduction. The gamma distribution with (positive) parameter $x$ has density with respect to Lebesgue measure on $(0, \infty)$ given by $e^{-t} t^{x-1} / \Gamma(x)$. The median $m(x)$ of this distribution is defined implicitly as

$$
\int_{0}^{m(x)} \frac{e^{-t} t^{x-1}}{\Gamma(x)} d t=\frac{1}{2}
$$

or

$$
\int_{0}^{m(x)} e^{-t} t^{x-1} d t=\frac{1}{2} \int_{0}^{\infty} e^{-t} t^{x-1} d t
$$

This is of course equivalent to

$$
\int_{m(x)}^{\infty} e^{-t} t^{x-1} d t=\frac{1}{2} \int_{0}^{\infty} e^{-t} t^{x-1} d t
$$

We show that $m$ is continuous and increasing. This is a consequence of a result about general convolution semigroups of probabilities on the positive half-line, that is given in Section 2. There we also show that $m$ is real analytic and that $m$ satisfies a certain differential equation.

We shall mainly study $m$ through the function

$$
\varphi(x) \equiv \log \frac{x}{m(x)}, \quad x>0 .
$$

This function appears if we make the substitution $u=\log (x / t)$ in the relation (2):

$$
\int_{-\infty}^{\varphi(x)} e^{-x\left(e^{-u}+u\right)} d u=\frac{1}{2} \int_{-\infty}^{\infty} e^{-x\left(e^{-u}+u\right)} d u
$$

Chen and Rubin (see [7]) studied the median of the gamma distribution and proved that $x-1 / 3<m(x)<x$ for $x>0$. The relation (4) was also used in [7] to

\footnotetext{
*Received October 29, 2004; accepted for publication August 24, 2006.

${ }^{\dagger}$ Department of Mathematics, University of Copenhagen, Universitetsparken 5, DK-2100, Copenhagen, Denmark (berg@math.ku.dk).

${ }_{\ddagger}^{\ddagger}$ Department of Natural Sciences, Royal Veterinary and Agricultural University, Thorvaldsensvej 40, DK-1871, Copenhagen, Denmark (henrikp@dina.kvl.dk). Research supported by the Carlsberg Foundation.
} 
establish that $\varphi(x)>0$, or equivalently $m(x)<x$. (It follows by observing that $\int_{-\infty}^{0} e^{-x\left(e^{-u}+u\right)} d u<\int_{0}^{\infty} e^{-x\left(e^{-u}+u\right)} d u$, which is true because $\sinh u>u$ for $u>0$.)

Chen and Rubin furthermore conjectured that the sequence $m(n)-n$ decreases. This conjecture has recently been verified by Alm [2]. See also Adell and Jodrá [1], which contains a simpler proof. We also mention Alzer [3], who proved that $m(n+1)-\alpha n$ decreases for all $n \geq 0$ exactly when $\alpha \geq 1$ and increases exactly when $\alpha \leq m(2)-\log 2$. (Note that $m(1)=\log 2$.)

In this paper we investigate the properties of $m$ as a function on $(0, \infty)$. Chen and Rubin's conjecture follows from the relation

$$
m^{\prime}(x)<1 \text { for all } x>0
$$

and it is our main goal to verify this relation. (See Theorem 3.4.)

The graph of $m$ can be found in Figure 1. (The dotted line is given by $x-1 / 3$.)

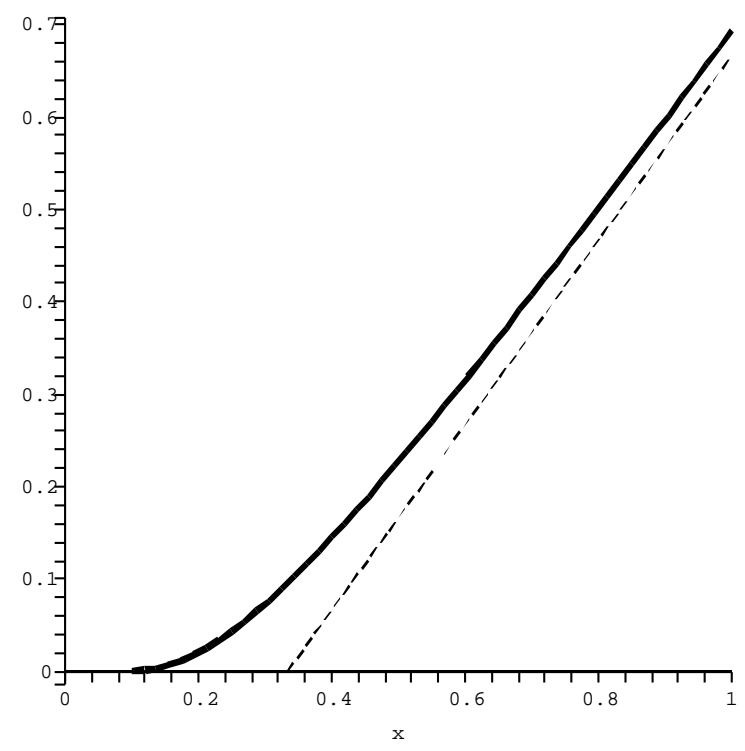

FIG. 1. The graph of $m$

In terms of $\varphi$ the inequality (5) takes the form $1-x \varphi^{\prime}(x)<e^{\varphi(x)}$. As a crucial step towards this result we show that $x \varphi(x)$ decreases and that

$$
\frac{1}{3}<x \varphi(x)<\log 2
$$

for all $x>0$ (see Proposition 3.6). In terms of $m$ this relation can be rewritten as

$$
x e^{-\log 2 / x}<m(x)<x e^{-1 / 3 x} .
$$

If we use that $e^{-a}<1-a+a^{2} / 2$ for $a>0$ then in fact

$$
m(x)<x\left(1-\frac{1}{3 x}+\frac{1}{18 x^{2}}\right)=x-\frac{1}{3}+\frac{1}{18 x}
$$


for all $x>0$. This result improves a result of Choi about $m(n+1)$, see $[8$, Theorem $1]$, even though it is there claimed to be best possible.

The asymptotic behaviour of $m(x)$ for $x \rightarrow 0$ and for $x \rightarrow \infty$ is given in Section 4. From this we can deduce that $m^{(k)}(x)>0$ for $x$ close to 0 for each $k$ while $(-1)^{k} m^{(k)}(x)>0$ for $x$ sufficiently large and $k \geq 2$. It is reasonable to believe that $m^{\prime \prime}(x)>0$ for all $x>0$, i.e. $m$ is convex, but the higher derivatives of odd order change sign.

2. Medians of convolution semigroups on the half-line. A family $\left\{\mu_{x}\right\}_{x>0}$ of probabilities concentrated on $[0, \infty)$ is called a convolution semigroup if it has the properties

(i) $\mu_{x}([0, \infty))=1$ for all $x>0$;

(ii) $\mu_{x} * \mu_{y}=\mu_{x+y}$ for all $x, y>0$;

(iii) $\mu_{x} \rightarrow \delta_{0}$ for $x \rightarrow 0$ in the vague topology (here $\delta_{0}$ denotes the Dirac mass at zero).

A probability measure $\mu$ on $[0, \infty)$ has median $m$ if

$$
\mu([0, m])=\frac{1}{2} .
$$

Of course a probability measure may not have a median and if it exists it may not be unique. However, if the measure has density w.r.t. Lebesgue measure on $[0, \infty)$ then the median exists: this follows from the fact that $M \rightarrow \mu([0, M])$ is continuous and increases from 0 to 1 . If the density is strictly positive almost everywhere on $[0, \infty)$ then the median is unique.

Proposition 2.1. Let $\left\{\mu_{x}\right\}_{x>0}$ be a convolution semigroup of probabilities on $[0, \infty)$ having a.e. strictly positive densities w.r.t. Lebesgue measure on $[0, \infty)$. Then the median $m(x)$ of $\mu_{x}$ is a continuous and strictly increasing function on $(0, \infty)$. Furthermore, $\lim _{x \rightarrow 0} m(x)=0$ and $\lim _{x \rightarrow \infty} m(x)=\infty$.

Proof. We write $d \mu_{x}(t)=g_{x}(t) d t$. As was noticed above, $m$ exists as a function on the positive half-line. We now show that $m$ is strictly increasing. Let $x>0$ and $h>0$. Then

$$
\begin{aligned}
\int_{0}^{m(x)} g_{x+h}(t) d t & =\int_{0}^{m(x)} g_{x} * g_{h}(t) d t \\
& =\int_{0}^{m(x)} \int_{0}^{t} g_{h}(t-s) g_{x}(s) d s d t \\
& =\int_{0}^{m(x)} \int_{s}^{m(x)} g_{h}(t-s) d t g_{x}(s) d s \\
& =\int_{0}^{m(x)} \int_{0}^{m(x)-s} g_{h}(t) d t g_{x}(s) d s \\
& <\int_{0}^{m(x)} \int_{0}^{\infty} g_{h}(t) d t g_{x}(s) d s \\
& =\int_{0}^{m(x)} g_{x}(s) d s=\frac{1}{2}
\end{aligned}
$$

Since $m(x+h)$ by definition satisfies

$$
\int_{0}^{m(x+h)} g_{x+h}(t) d t=\frac{1}{2},
$$


it follows that $m(x)<m(x+h)$. Therefore $m$ is strictly increasing.

Concerning the continuity we first notice that for any $A>0$,

$$
\int_{0}^{A} g_{x_{0}+h}(t) d t \rightarrow \int_{0}^{A} g_{x_{0}}(t) d t
$$

as $h \rightarrow 0$. In fact, by the same computation as above,

$$
\begin{aligned}
\int_{0}^{A} g_{x_{0}+h}(t) d t & =\int_{0}^{A} g_{x_{0}} * g_{h}(t) d t \\
& =\int_{0}^{A} \int_{0}^{A-s} g_{h}(t) d t g_{x_{0}}(s) d s \\
& \rightarrow \int_{0}^{A} \int_{0}^{A-s} d \delta_{0}(t) g_{x_{0}}(s) d s \\
& =\int_{0}^{A} g_{x_{0}}(s) d s,
\end{aligned}
$$

as $h \rightarrow 0$, because of the vague convergence. (All measures in question have the same total mass, so vague convergence implies weak convergence.) Now let $x_{0}>0$ and let $\epsilon>0$ be given. For $A=m\left(x_{0}\right)+\epsilon$ we have, as $h \rightarrow 0_{+}$,

$$
\int_{0}^{m\left(x_{0}\right)+\epsilon} g_{x_{0}+h}(t) d t \rightarrow \int_{0}^{m\left(x_{0}\right)+\epsilon} g_{x_{0}}(t) d t>\frac{1}{2}
$$

so that $m\left(x_{0}+h\right) \leq m\left(x_{0}\right)+\epsilon$ for all sufficiently small and positive $h$. Similarly, $m\left(x_{0}-h\right) \geq m\left(x_{0}\right)-\epsilon$, and this shows that $m$ is continuous.

To show that $\lim _{x \rightarrow 0} m(x)=0$ and $\lim _{x \rightarrow \infty} m(x)=\infty$, it is enough to exclude that

(a) there is $a>0$ such that $m(x) \geq a$ for $x>0$, and

(b) there is $A>0$ such that $m(x) \leq A$ for $x>0$,

$m$ being increasing.

If (a) holds then

$$
\int_{0}^{a} g_{x}(t) d t \leq \int_{0}^{m(x)} g_{x}(t) d t=\frac{1}{2}
$$

for all $x$. Since $\mu_{x}$ converges vaguely to the Dirac mass at 0 for $x$ tending to 0 we get

$$
1=\delta_{0}([0, a)) \leq \liminf _{x \rightarrow 0} \mu_{x}([0, a)) \leq \frac{1}{2},
$$

which is a contradiction.

If (b) holds then

$$
\frac{1}{2}=\int_{0}^{m(x)} g_{x}(t) d t \leq \int_{0}^{A} g_{x}(t) d t \leq e^{A} \int_{0}^{A} e^{-t} g_{x}(t) d t<e^{A} e^{-x f(1)},
$$

where $f$ is the Bernstein function corresponding to the convolution semigroup (see [5]). However, $f(1)>0$ so that the right-hand side tends to 0 as $x \rightarrow \infty$. We have reached a contradiction. 
The gamma distributions $\left\{e^{-t} t^{x-1} / \Gamma(x) d t\right\}_{x>0}$ is an example of such a convolution semigroup. Other examples can be constructed from the one-sided stable distribution of order $\alpha(0<\alpha<1)$, and the log-normal distribution; see [14]. Both of these distributions are infinitely divisible and can be embedded in a convolution semigroup. The median of either of these semigroups has the properties of Proposition 2.1.

We now specialize to consider the median $m(x)$ of the gamma distributions. We notice

Proposition 2.2. The median $m(x)$ of the gamma distributions is real analytic.

Proof. We consider the $C^{1}$-function

$$
F(x, y)=\int_{0}^{y} e^{-t} t^{x-1} \frac{1}{\Gamma(x)} d t, \quad x, y>0 .
$$

The median is implicitly defined as $F(x, m(x))=1 / 2$. The fact that the continuous function $m$ is $C^{1}$ follows from the implicit function theorem, which yields the following differential equation for $m$ :

$$
e^{-m(x)} m(x)^{x-1} m^{\prime}(x)=\frac{1}{2} \Gamma^{\prime}(x)-\int_{0}^{m(x)}(\log t) e^{-t} t^{x-1} d t,
$$

which shows that $m$ satisfies a differential equation of the form

$$
m^{\prime}(x)=G(x, m(x)),
$$

with $G(x, y)$ being real analytic for $x, y>0$. Therefore $m$ is real analytic.

REMARK 2.3. From (6) it seems difficult to deduce monotonicity properties of $m$; e.g. it is not at all clear that $m^{\prime}(x)>0$, which follows from Proposition 2.1. For another derivation of $m^{\prime}(x)>0$ see Proposition 3.6.

3. Uniform results. In this section the generalized version of Chen and Rubin's conjecture (Theorem 3.4) is proved.

Proposition 3.3 below is the key to our results. Before stating it we need some notation.

We consider the function $f(x)=e^{-x}+x$. It is easily seen that $f(x)$ decreases for $x<0$ and then increases for $x>0$. Hence $f$ has an inverse on $(-\infty, 0]$, which we call $u$, and an inverse on $[0, \infty)$, which we call $v$. The function $u$ is defined for $t \geq 1$ as $u(t) \leq 0$ and

$$
e^{-u(t)}+u(t)=t, \quad t \geq 1
$$

the function $v$ as $v(t) \geq 0$ and

$$
e^{-v(t)}+v(t)=t, \quad t \geq 1 .
$$

The following function $\xi$ plays an important role:

$$
\xi(t)=\frac{1}{1-e^{-v(t)}}+\frac{1}{1-e^{-u(t)}}=u^{\prime}(t)+v^{\prime}(t) .
$$

Since $u(t) \rightarrow-\infty$ and $v(t) \rightarrow \infty$ for $t \rightarrow \infty$ it follows that

$$
\xi(t) \rightarrow 1 \quad \text { as } \quad t \rightarrow \infty .
$$


The function $\xi(t)$ is defined for $t>1$. For $t=1$ the expression (7) yields $\infty-\infty$ and a closer study is necessary.

Proposition 3.1. The function $\xi(t)$ is holomorphic in a neighbourhood of $t=1$ and $\xi(1)=2 / 3, \xi^{\prime}(1)=8 / 135$.

The monotonicity properties of $\xi$ also play an important role in our investigations. The following result holds.

Proposition 3.2. The function $\xi(t)$ is increasing and concave for $t>1$.

These two propositions are proved in Section 5. It is in fact proved that $\xi$ has a holomorphic extension to the cut plane $\mathbb{C} \backslash\{x \pm 2 \pi i \mid x \geq 1\}$ (Corollary 5.5). Furthermore, an integral representation of $\xi$ is obtained (Proposition 5.8) from which the monotonicity properties are derived. It is shown that $\xi(t)$ is increasing for $t \in \mathbb{R}$ and has the limit 0 as $t$ tends to $-\infty$. Hence the function cannot be concave on the whole real line and this is reflected in the technicalities establishing concavity on the interval $[1, \infty)$.

Proposition 3.3. We have

$$
2 \int_{0}^{\varphi(x)} e^{-x\left(e^{-u}+u\right)} d u=\int_{1}^{\infty} \xi(t) e^{-x t} d t
$$

where the function $\xi$ is defined in (7).

Proof. From (4) it follows

$$
2 \int_{0}^{\varphi(x)} e^{-x\left(e^{-u}+u\right)} d u=\int_{0}^{\infty} e^{-x\left(e^{-u}+u\right)} d u-\int_{-\infty}^{0} e^{-x\left(e^{-u}+u\right)} d u .
$$

In the first integral on the right-hand side we make the substitution $u=v(t)$ and in the second the substitution $u=u(t)$, where $u(t)$ and $v(t)$ denote the functions above. This leads to

$$
\begin{aligned}
2 \int_{0}^{\varphi(x)} e^{-x\left(e^{-u}+u\right)} d u & =\int_{1}^{\infty} e^{-x t}\left(\frac{1}{1-e^{-v(t)}}+\frac{1}{1-e^{-u(t)}}\right) d t \\
& =\int_{1}^{\infty} \xi(t) e^{-x t} d t .
\end{aligned}
$$

As mentioned in the introduction the inequality $m^{\prime}(x)<1$ follows from the result below.

Theorem 3.4. The inequality

$$
1-x \varphi^{\prime}(x)<e^{\varphi(x)}
$$

holds for all $x>0$.

Before proving this result, we give the following Lemma.

LEMMA 3.5. The relation

$$
2 \int_{0}^{x \varphi(x)} e^{-s} e^{x\left(1-e^{-s / x}\right)} d s=\frac{2}{3}+\int_{1}^{\infty} \xi^{\prime}(t) e^{-x(t-1)} d t
$$

holds for all $x>0$. 
Proof. On the left hand side of the relation in Proposition 3.3 we make the substitution $s=x u$ and get in this way

$$
\frac{2}{x} \int_{0}^{x \varphi(x)} e^{-s-x e^{-s / x}} d s=\int_{1}^{\infty} \xi(t) e^{-x t} d t
$$

hence by integration by parts

$$
\begin{aligned}
2 \int_{0}^{x \varphi(x)} e^{-s-x e^{-s / x}} d s & =\int_{1}^{\infty} \xi(t) x e^{-x t} d t \\
& =e^{-x} \xi(1)+\int_{1}^{\infty} \xi^{\prime}(t) e^{-x t} d t .
\end{aligned}
$$

Here $\xi(1)=2 / 3$ (see Proposition 3.1) and the assertion of the lemma now follows by multiplication by $e^{x}$.

Proposition 3.6. The function $x \rightarrow x \varphi(x)$ decreases for $x>0$ and

$$
\begin{aligned}
& \lim _{x \rightarrow 0_{+}} x \varphi(x)=\log 2, \\
& \lim _{x \rightarrow \infty} x \varphi(x)=\frac{1}{3} .
\end{aligned}
$$

In particular $\varphi$ is decreasing from $\infty$ to 0 (and $m$ is increasing).

Proof. It follows from Lemma 3.5, by differentiation, that

$$
\begin{aligned}
& e^{-x\left(\varphi(x)-1+e^{-\varphi(x)}\right)}(x \varphi(x))^{\prime}= \\
& -\int_{0}^{x \varphi(x)} e^{-s} e^{x\left(1-e^{-s / x}\right)}\left(1-\left(1+\frac{s}{x}\right) e^{-s / x}\right) d s \\
& -\frac{1}{2} \int_{0}^{\infty} t e^{-x t} \xi^{\prime}(t+1) d t .
\end{aligned}
$$

Since $1-(1+a) e^{-a}>0$ for $a>0$ and $\xi^{\prime}(t+1)>0$ for $t>0$ (see Proposition 3.2) we have

$$
A(x) \equiv \int_{0}^{x \varphi(x)} e^{-s} e^{x\left(1-e^{-s / x}\right)}\left(1-\left(1+\frac{s}{x}\right) e^{-s / x}\right) d s>0
$$

and

$$
B(x) \equiv \frac{1}{2} \int_{0}^{\infty} t e^{-x t} \xi^{\prime}(t+1) d t>0
$$

Therefore

$$
(x \varphi(x))^{\prime}=-e^{x\left(\varphi(x)-1+e^{-\varphi(x)}\right)}(A(x)+B(x)),
$$

which is a negative quantity, so $x \varphi(x)$ decreases.

Furthermore, since $e^{-s} e^{x\left(1-e^{-s / x}\right)} \geq e^{-s}$, we get from Lemma 3.5,

$$
2 \int_{0}^{x \varphi(x)} e^{-s} d s \leq \frac{2}{3}+\int_{1}^{\infty} \xi^{\prime}(t) d t=\frac{2}{3}+\lim _{x \rightarrow \infty} \xi(x)-\xi(1)=1,
$$


since $\lim _{x \rightarrow \infty} \xi(x)=1$ (see (8)). Therefore $x \varphi(x)$ is bounded by $\log 2$ for all $x>0$.

Let $l=\lim _{x \rightarrow 0_{+}} x \varphi(x)$. Then by the dominated convergence theorem we find

$$
2 \int_{0}^{l} e^{-s} d s=\frac{2}{3}+\int_{1}^{\infty} \xi^{\prime}(t) d t=1
$$

so that $l=\log 2$.

Finally, let $L=\lim _{x \rightarrow \infty} x \varphi(x)$. In the same way, we get

$$
2 \int_{0}^{L} 1 d s=\frac{2}{3}
$$

so that $L=1 / 3$.

Proof of Theorem 3.4. With the expressions $A(x)$ and $B(x)$ and the relation (10) from the proof of Proposition 3.6 we obtain

$$
\begin{aligned}
1-x \varphi^{\prime}(x) & =e^{x\left(\varphi(x)-1+e^{-\varphi(x)}\right.}(A(x)+B(x))+\varphi(x)+1 \\
& \leq e^{x \varphi(x)}(A(x)+B(x))+\varphi(x)+1 .
\end{aligned}
$$

Now, using that $1-e^{-a}<a$ and $1-(1+a) e^{-a}<a^{2} / 2$ for $a>0$, it follows that

$$
A(x) \leq \int_{0}^{x \varphi(x)} e^{-s} e^{x(s / x)}\left(\frac{s^{2}}{2 x^{2}}\right) d s=\frac{x \varphi(x)^{3}}{6} .
$$

Furthermore, since $\xi^{\prime}(t+1) \leq \xi^{\prime}(1)=8 / 135$ for $t \geq 0$,

$$
B(x) \leq \frac{1}{2} \frac{8}{135} \int_{0}^{\infty} t e^{-x t} d t=\frac{4}{135 x^{2}} .
$$

This gives

$$
1-x \varphi^{\prime}(x) \leq 1+\varphi(x)+e^{x \varphi(x)}\left(\frac{x \varphi(x)}{6}+\frac{4}{135(x \varphi(x))^{2}}\right) \varphi(x)^{2} .
$$

It is easily seen that the function

$$
\rho(u)=e^{u}\left(\frac{u}{6}+\frac{4}{135 u^{2}}\right)
$$

attains its maximum on $[1 / 3, \log 2]$ at $u=1 / 3$ with value

$$
\rho\left(\frac{1}{3}\right)=\frac{29}{90} e^{1 / 3}<\frac{1}{2} .
$$

We obtain from this the relation

$$
1-x \varphi^{\prime}(x)<1+\varphi(x)+\frac{1}{2} \varphi(x)^{2}<e^{\varphi(x)} .
$$

REMARK 3.7. The difference between $e^{\varphi(x)}$ and $1+\varphi(x)+\frac{1}{2} \varphi(x)^{2}$ is $O\left(\varphi(x)^{3}\right)$, that is (by Proposition 3.6) the difference is $O\left(x^{-3}\right)$. Hence for large $x$ the difference is very small, reflecting the fact that $m^{\prime}(x)$ is very close to 1 . For $x$ close to 0 , the 
difference is large, reflecting the fact that $m^{\prime}(x)$ approaches 0 rapidly. See Proposition 4.1.

Remark 3.8. Defining $\theta(n)$ for natural numbers $n$ by

$$
\frac{e^{n}}{2}=\sum_{k=0}^{n-1} \frac{n^{k}}{k !}+\theta(n) \frac{n^{n}}{n !},
$$

Ramanujan [13] claimed that $\frac{1}{3}<\theta(n)<\frac{1}{2}$. This was later proved independently by Szegö [15] and Watson [16]. Further details about Ramanujan's problem can be found in [2], [6], [10] and [8].

Watson obtained the relation

$$
\theta(n)=1+\frac{n}{2}\left(\int_{0}^{1}\left(t e^{1-t}\right)^{n} d t-\int_{1}^{\infty}\left(t e^{1-t}\right)^{n} d t\right)
$$

and noticed that the right-hand side of this equation makes perfect sence when $n$ is replaced by a positive real variable $x$. The substitution $t=e^{-u}$ in the integrals and some computation lead to

$$
\theta(x)=\frac{x}{2} \int_{0}^{\infty} e^{-x t} \xi(t+1) d t .
$$

The inequalities $1 / 3<\theta(x)<1 / 2$ can now be obtained from the inequalities $2 / 3<$ $\xi(t+1)<1$.

4. Asymptotic results. Here the behaviour of $m$ and $m^{\prime}$ at zero and at infinity is described. We use $f(x) \sim g(x)$ for $x \rightarrow 0$ to denote that

$$
\lim _{x \rightarrow 0} f(x) / g(x)=1 .
$$

Proposition 4.1. We have $m(x)^{x} \rightarrow 1 / 2$ as $x \rightarrow 0$ and

$$
m(x) \sim e^{-\gamma} 2^{-1 / x} \quad \text { as } \quad x \rightarrow 0 .
$$

Furthermore,

$$
m^{\prime}(x) \sim(\log 2) e^{-\gamma} \frac{2^{-1 / x}}{x^{2}} \quad \text { as } \quad x \rightarrow 0 .
$$

(The constant $\gamma$ denotes Euler's constant.)

Proof. Let $l(x)=\log \left(m(x)^{x}\right)=-x \varphi(x)+x \log x$. From Proposition 3.6 it follows that $l(x) \rightarrow-\log 2$ as $x \rightarrow 0$. Therefore $m(x)^{x} \rightarrow 1 / 2$ as $x \rightarrow 0$.

By the definition of $m(x)$ and by the functional equation of the gamma function we have

$$
\int_{0}^{m(x)} e^{-t} x t^{x-1} d t=\frac{1}{2} \Gamma(x+1) .
$$

Integration by parts on the integral on the left hand side in this relation yields

$$
m(x)^{x} e^{-m(x)}+\int_{0}^{m(x)} e^{-t} t^{x} d t=\frac{1}{2} \Gamma(x+1) .
$$


We next differentiate this relation and get after some manipulation

$$
\log m(x)+\frac{x}{m(x)} m^{\prime}(x)=e^{m(x)} m(x)^{-x}\left(\frac{1}{2} \Gamma^{\prime}(x+1)-\int_{0}^{m(x)}(\log t) e^{-t} t^{x} d t\right) .
$$

Since $m(x)^{x} \rightarrow 1 / 2$ as $x \rightarrow 0$ it follows that

$$
\lim _{x \rightarrow 0}\left(\log m(x)+\frac{x}{m(x)} m^{\prime}(x)\right)=\Gamma^{\prime}(1)=-\gamma .
$$

This is the same as $l^{\prime}(x)=\left(\log \left(m(x)^{x}\right)\right)^{\prime} \rightarrow-\gamma$ as $x \rightarrow 0$. Using l'Hospital's rule we get

$$
\frac{l(x)+\log 2}{x} \rightarrow-\gamma
$$

for $x \rightarrow 0$. Therefore $m(x) \sim 2^{-1 / x} e^{-\gamma}$ as $x \rightarrow 0$.

From (12) it follows that

$$
x \log m(x)+\frac{x^{2}}{m(x)} m^{\prime}(x) \rightarrow 0
$$

for $x \rightarrow 0$ and, since (as used before) $x \log m(x) \rightarrow-\log 2$, we get

$$
m^{\prime}(x) \sim \frac{\log 2}{x^{2}} 2^{-1 / x} e^{-\gamma}
$$

as $x \rightarrow 0$.

Proposition 4.2. The functions $m$ and $m^{\prime}$ have asymptotic expansions at infinity. We have in particular

$$
m^{\prime}(x)=1-\frac{8}{405 x^{2}}-\frac{368}{25515 x^{3}}+o\left(x^{-3}\right) \quad \text { as } \quad x \rightarrow \infty
$$

and

$$
m(x)=x-\frac{1}{3}+\frac{8}{405 x}+\frac{184}{25515 x^{2}}+o\left(x^{-2}\right) \quad \text { as } \quad x \rightarrow \infty .
$$

REMARK 4.3. Choi [8] found the asymptotic expansion of $m(n+1)$ up to order $o\left(n^{-3}\right)$. Higher order expansions of $m(n+1)$ were found in [11]. In the appendix we have included higher order expansions of $m(x)$ and $\varphi(x)$ (and higher order derivatives of the function $\xi$ at 1). Because of the complexity, the computations behind the expansions in the appendix were made with "Maple 9", using the same method as described in Lemma 4.5.

Some properties of $\xi$ are also needed here, namely:

Lemma 4.4. For any $n \geq 1, \lim _{t \rightarrow \infty} \xi^{(n)}(t)=0$.

This lemma is proved in the last part of Section 5 .

LEMma 4.5. The functions $\varphi$ and $\varphi^{\prime}$ have asymptotic expansions at infinity. In particular

$$
\varphi(x)=\frac{1}{3 x}+\frac{29}{810 x^{2}}-\frac{37}{25515 x^{3}}+o\left(x^{-3}\right)
$$


as $x \rightarrow \infty$.

Proof. We have already seen in Proposition 3.6 that $\varphi(x)=O\left(x^{-1}\right)$. Therefore

$$
2 \int_{0}^{\varphi(x)} e^{-x\left(u+e^{-u}-1\right)} d u=2 \sum_{k=0}^{n} \frac{(-1)^{k} x^{k}}{k !} \int_{0}^{\varphi(x)}\left(u+e^{-u}-1\right)^{k} d u+o\left(x^{-(n+1)}\right) .
$$

On the other hand, by partial integration and Lemma 4.4,

$$
\int_{0}^{\infty} \xi(t+1) e^{-x t} d t=\sum_{k=0}^{n} \frac{\xi^{(k)}(1)}{x^{k+1}}+o\left(x^{-(n+1)}\right)
$$

From Proposition 3.3 we thus get

$$
\varphi(x)=\sum_{k=0}^{n} \frac{\xi^{(k)}(1)}{2 x^{k+1}}-\sum_{k=1}^{n} \frac{(-1)^{k} x^{k}}{k !} \int_{0}^{\varphi(x)}\left(u+e^{-u}-1\right)^{k} d u+o\left(x^{-(n+1)}\right) .
$$

From this relation it is possible to deduce that $\varphi(x)$ has an asymptotic expansion of the form

$$
\varphi(x)=\sum_{k=1}^{n} \frac{c_{k}}{x^{k}}+o\left(x^{-n}\right)
$$

as $x \rightarrow \infty$ and for any $n$. (See below.)

Let us first find the coefficients $c_{1}, c_{2}$ and $c_{3}$ in the expansion. If $n=0$ the relation (14) yields, since $\xi(1)=2 / 3$,

$$
\varphi(x)=\frac{1}{3 x}+o\left(x^{-1}\right)
$$

(This was already found in Proposition 3.6.) For $n=1$ we get, using $\xi^{\prime}(1)=8 / 135$,

$$
\varphi(x)=\frac{1}{3 x}+\frac{4}{135 x^{2}}+x \int_{0}^{\varphi(x)}\left(u+e^{-u}-1\right) d u+o\left(x^{-2}\right) .
$$

Now, $u+e^{-u}-1=u^{2} / 2+o\left(u^{2}\right)$ for $u \rightarrow 0$ and therefore

$$
\varphi(x)=\frac{1}{3 x}+\frac{4}{135 x^{2}}+x\left(\frac{\varphi(x)^{3}}{6}+o\left(x^{-3}\right)\right)+o\left(x^{-2}\right) .
$$

Then we use the result for $n=0$ to obtain that $x \varphi(x)^{3}=1 /\left(27 x^{2}\right)+o\left(x^{-2}\right)$, and if we insert this into the relation above it follows that

$$
\varphi(x)=\frac{1}{3 x}+\left(\frac{4}{135}+\frac{1}{162}\right) \frac{1}{x^{2}}+o\left(x^{-2}\right)=\frac{1}{3 x}+\frac{29}{810 x^{2}}+o\left(x^{-2}\right) .
$$

The argument is repeated and yields the following for $n=2$ :

$$
\begin{aligned}
\varphi(x)= & \frac{1}{3 x}+\frac{4}{135 x^{2}}-\frac{8}{2835 x^{3}} \\
& +x \int_{0}^{\varphi(x)}\left(u+e^{-u}-1\right) d u-\frac{x^{2}}{2} \int_{0}^{\varphi(x)}\left(u+e^{-u}-1\right)^{2} d u+o\left(x^{-3}\right) .
\end{aligned}
$$


Here we use $u+e^{-u}-1=u^{2} / 2-u^{3} / 6+o\left(u^{3}\right)$ and $\left(u+e^{-u}-1\right)^{2}=u^{4} / 4+o\left(u^{4}\right)$ for $u \rightarrow 0$ and get

$$
\begin{aligned}
\varphi(x)= & \frac{1}{3 x}+\frac{4}{135 x^{2}}-\frac{8}{2835 x^{3}} \\
& +x\left(\frac{\varphi(x)^{3}}{6}-\frac{\varphi(x)^{4}}{24}+o\left(x^{-4}\right)\right) \\
& -\frac{x^{2}}{2}\left(\frac{\varphi(x)^{5}}{20}+o\left(x^{-5}\right)\right)+o\left(x^{-3}\right) .
\end{aligned}
$$

In the term $x \varphi(x)^{3} / 6$ the expansion of $\varphi(x)$ for $n=1$ is substituted and in the terms $x \varphi(x)^{4} / 24$ and $x^{2} \varphi(x)^{5} / 40$ the expansion of $\varphi(x)$ for $n=0$. In this way we get

$$
\begin{aligned}
\varphi(x) & =\frac{1}{3 x}+\frac{29}{810 x^{2}}+\left(-\frac{8}{2835}+\frac{1}{18} \frac{29}{810}-\frac{1}{24} \frac{1}{81}-\frac{1}{40} \frac{1}{243}\right) \frac{1}{x^{3}}+o\left(x^{-3}\right) \\
& =\frac{1}{3 x}+\frac{29}{810 x^{2}}-\frac{37}{25515 x^{3}}+o\left(x^{-3}\right) .
\end{aligned}
$$

These computations also indicate how to show that there is an asymptotic expansion (15) for every $n \geq 1$. One could use an inductive argument based on the relation (14): First of all, the sum

$$
\sum_{k=0}^{n} \frac{\xi^{(k)}(1)}{2 x^{k+1}}
$$

contains a term of order $1 / x^{n+1}$. Next the integrand $\left(u+e^{-u}-1\right)^{k}$ is approximated by its Taylor polynomial of order $n+k$,

$$
\left(u+e^{-u}-1\right)^{k}=\sum_{l=2 k}^{n+k} \alpha_{k, l} u^{l}+o\left(u^{n+k}\right),
$$

and the expansion $\varphi(x)=\sum_{k=1}^{n} c_{k} / x^{-k}+o\left(x^{-n}\right)$ is then used in the upper limit of the integrals in the sum

$$
\sum_{k=1}^{n} \frac{(-1)^{k} x^{k}}{k !} \int_{0}^{\varphi(x)}\left(u+e^{-u}-1\right)^{k} d u .
$$

Using these approximations it is possible to obtain

$$
\varphi(x)=\sum_{k=0}^{n} \frac{\xi^{(k)}(1)}{2 x^{k+1}}-\sum_{k=1}^{n} \frac{(-1)^{k} x^{k}}{k !} \sum_{l=2 k}^{n+k} \frac{\alpha_{k, l}}{l+1}\left(\sum_{j=1}^{n} \frac{c_{j}}{x^{j}}\right)^{l+1}+o\left(x^{-(n+1)}\right) .
$$

This is an expansion of $\varphi(x)$ of order $n+1$.

To see that also $\varphi^{\prime}(x)$ has an asymptotic expansion we differentiate (9) and get

$$
2 \varphi^{\prime}(x) e^{-x\left(\varphi(x)+e^{-\varphi(x)}\right)}-2 \int_{0}^{\varphi(x)} e^{-x\left(u+e^{-u}\right)}\left(u+e^{-u}\right) d u=-\int_{1}^{\infty} t \xi(t) e^{-x t} d t .
$$

Adding (9) to the relation above we get after multiplication by $e^{x}$ and a change of variable $s=t-1$,

$$
\begin{aligned}
& 2 \varphi^{\prime}(x) e^{-x\left(\varphi(x)+e^{-\varphi(x)}-1\right)} \\
& =2 \int_{0}^{\varphi(x)} e^{-x\left(u+e^{-u}-1\right)}\left(u+e^{-u}-1\right) d u-\int_{0}^{\infty} s \xi(1+s) e^{-x s} d s .
\end{aligned}
$$


In the first integral we use again Taylor approximation and in the second integral integration by parts. Using also the asymptotic expansion of $e^{x\left(\varphi(x)+e^{-\varphi(x)}-1\right)}$ it follows finally that there is an asymptotic expansion of $\varphi^{\prime}(x)$. The coefficients in this expansion can be identified by integrating the expansion and using the known expansion of $\varphi(x)$, cf [4][Appendix C].

Proof of Proposition 4.2. Since $\varphi(x)$ has an asymptotic expansion,

$$
m(x)=x e^{-\varphi(x)}=x \sum_{k=0}^{\infty} \frac{(-1)^{k} \varphi(x)^{k}}{k !}
$$

also has an asymptotic expansion and in particular

$$
m(x)=x e^{-\varphi(x)}=x\left(1-\varphi(x)+\frac{\varphi(x)^{2}}{2}-\frac{\varphi(x)^{3}}{6}+o\left(x^{-3}\right)\right) .
$$

We insert in this relation the asymptotic expansion of $\varphi(x)$ from the lemma above and get, after some computation,

$$
\begin{aligned}
m(x) & =x\left(1-\frac{1}{3 x}+\frac{8}{405 x^{2}}+\frac{184}{25515 x^{3}}+o\left(x^{-3}\right)\right) \\
& =x-\frac{1}{3}+\frac{8}{405 x}+\frac{184}{25515 x^{2}}+o\left(x^{-2}\right) .
\end{aligned}
$$

Since $\varphi^{\prime}(x)$ has an asymptotic expansion, the same is true for $m^{\prime}(x)$, since $m^{\prime}(x)=$ $\left(1-x \varphi^{\prime}(x)\right) e^{-\varphi(x)}$. The expansion of $m(x)$ can be found by integrating the expansion of $m^{\prime}(x)$, and this gives the desired expansion of $m^{\prime}(x)$.

REMARK 4.6. It is clear that the methods above can be continued so the asymptotic behaviour of $m^{(k)}(x), k \geq 2$ for $x \rightarrow 0$ and $x \rightarrow \infty$ can be determined by differentiation of the asymptotic formulas for $m^{\prime}(x)$.

REMARK 4.7. The asymptotic expansion of Ramanujans function $\theta(x)$

$$
\theta(x)=\sum_{k=0}^{n} \frac{\xi^{(k)}(1)}{2 x^{k}}+o\left(x^{-n}\right)
$$

follows from (13) and (11).

5. Properties of the auxiliary function $\xi$. In this section the properties of the function $\xi$, stated in the propositions 3.1, 3.2 and in Lemma 4.4 are derived. The monotonicity properties of $\xi$ we found surprisingly difficult to establish. Much of the difficulty lies in the fact that $\xi$ is given as a sum of two terms, where it is necessary to control the cancellation between these two terms.

\section{Behaviour of $\xi$ at $t=1$.}

Proof of Proposition 3.1. We put $f(z)=e^{-z}+z$ and notice that the function $f(z)-1$ has a zero of multiplicity 2 at $z=0$. Hence there exists a holomorphic function $h$ in a neighbourhood of 0 such that $f(z)-1=h(z)^{2}$ there. Since $h^{\prime}(0)^{2}=1 / 2 \neq 0, h$ is one-to-one near $z=0$. We choose $h^{\prime}(0)=1 / \sqrt{2}$ and with this choice $h$ is uniquely determined. 
There is thus a radius $r>0$ such that for any $w$ with $|w|<r$, there are exactly two solutions to the equation $f(z)=w$, namely $z=h^{-1}( \pm \sqrt{w-1})$. In particular, for $t>1$ and close to 1 we have

$$
u(t)=h^{-1}(-\sqrt{t-1}) \text { and } v(t)=h^{-1}(\sqrt{t-1}),
$$

where $u$ and $v$ are the functions appearing in (7). Denoting the Taylor series of $h^{-1}(w)$ by $\sum_{n=1}^{\infty} a_{n} w^{n}$ it follows that

$$
u(t)+v(t)=\sum_{k=1}^{\infty} 2 a_{2 k}(t-1)^{k} .
$$

Since $\xi(t)=u^{\prime}(t)+v^{\prime}(t)$, we find $\xi(1)=2 a_{2}$ and $\xi^{\prime}(1)=4 a_{4}$. The numbers $a_{2}$ and $a_{4}$ can be found as follows. If $h(z)=\sum_{n=1}^{\infty} b_{n} z^{n}$ then

$$
\left(\sum_{n=1}^{\infty} b_{n} z^{n}\right)^{2}=e^{-z}+z-1=\frac{1}{2} z^{2}-\frac{1}{6} z^{3}+\frac{1}{24} z^{4}+\ldots
$$

and this gives after some manipulation $b_{1}=1 / \sqrt{2}, b_{2}=-\sqrt{2} / 12, b_{3}=\sqrt{2} / 72$ and $b_{4}=-\sqrt{2} / 540$. The numbers $a_{2}$ and $a_{4}$ can now be identified if we differentiate four times the relation $\left(h^{-1}\right)(h(z))=z$ and put $z=0$ (so that expressions involving e.g. $\left(h^{-1}\right)^{(4)}(0)$ appear). This gives, again after some manipulation, $\left(h^{-1}\right)^{(2)}(0)=2 / 3$ and $\left(h^{-1}\right)^{(4)}(0)=16 / 45$, so that $\xi(1)=2 a_{2}=2 / 3$ and $\xi^{\prime}(1)=4 a_{4}=4 \cdot(16 / 45) \cdot(1 / 24)=$ $8 / 135$.

REMARK 5.1. One can in principle find any derivative of $\xi$ at 1 in this way. Higher order derivatives of $\xi$ at 1 can be found in the appendix.

Monotonicity properties of $\xi$. The monotonicity properties are derived through an approach using complex analysis and we shall make extensive use of the theory of the so-called Pick functions, see [9]. A holomorphic function $p$ defined in the upper half plane is a Pick function if it maps the upper half plane into the closed upper half plane, or put in another way, if $\Im p$ is a non-negative harmonic function. A Pick function has an integral representation,

$$
p(z)=a z+b+\int_{-\infty}^{\infty}\left(\frac{1}{t-z}-\frac{t}{t^{2}+1}\right) d \mu(t),
$$

where $a \geq 0, b$ is real and $\mu$ is a positive measure on the real line. Furthermore,

$$
a=\lim _{y \rightarrow \infty} \frac{\Im p(i y)}{y}, \quad b=\Re p(i)
$$

and

$$
\mu=\lim _{y \rightarrow 0_{+}} \frac{\Im p(x+i y)}{\pi} d x
$$

in the vague topology. Note that a Pick function can be extended to a holomorphic function in $\mathbb{C} \backslash \mathbb{R}$ by (16). If $A \subseteq \mathbb{R}$ is closed we recall that $p$ has a holomorphic extension to $\mathbb{C} \backslash A$ if and only if the support of $\mu$ is contained in $A$. 
The proof of the monotonicity properties of $\xi$ (Proposition 3.2) is based on an investigation of the conformal properties of the holomorphic function

$$
f(z)=e^{-z}+z .
$$

The key to the proof is an integral representation of $\xi$ that is given in Proposition 5.8. Some quite technical lemmas are needed, basically to exploit the relation between the functions $f$ and $\xi$.

LEMmA 5.2. The function $f(z)=e^{-z}+z$ is a conformal mapping of the strip

$$
\mathcal{S}_{2 \pi}=\{0<\Im z<2 \pi\}
$$

onto the domain

$$
\mathcal{T}=\mathbb{C} \backslash(\{\Im w=2 \pi, \Re w \geq 1\} \cup\{\Im w=0, \Re w \geq 1\}) .
$$

The image of some horizontal lines in $\mathcal{S}_{2 \pi}$ under the function $f$ is shown in Figure 2 .

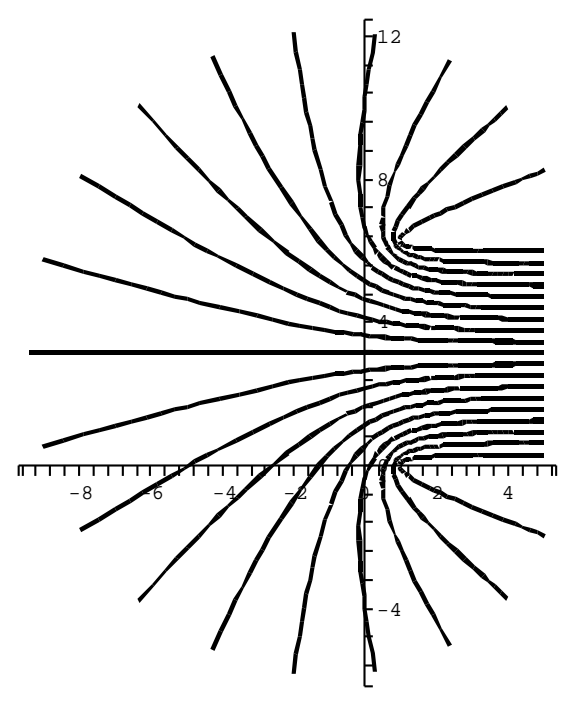

FIG. 2. The image of horizontal lines under $f(z)=e^{-z}+z$.

Proof. We have $f(x+i y)=\sigma+i \tau$ if and only if

$$
\begin{aligned}
e^{-x} \cos y+x & =\sigma \\
-e^{-x} \sin y+y & =\tau .
\end{aligned}
$$

If $y=\pi$ then $\tau=\pi$ and $\sigma=x-e^{-x}$ so $f$ maps the horizontal line $\mathbb{R}+i \pi$ onto itself and is one-to-one there.

Note that if $0<y<\pi$ then $\tau=-e^{-x} \sin y+y<y$ so

$$
f(\{0<\Im z<\pi\}) \subseteq\{\Im w<\pi\} .
$$


For $\tau<\pi$ we get $e^{x}=(\sin y) /(y-\tau)$ and since $\tau<y$ this gives $x=\log (\sin y /(y-$ $\tau))$. Therefore we shall look for a solution $y$ to the equation

$$
\log \frac{\sin y}{y-\tau}+\frac{(y-\tau) \cos y}{\sin y}=\sigma .
$$

For $\tau<\pi$ we put

$$
F_{\tau}(y)=\log \frac{\sin y}{y-\tau}+\frac{(y-\tau) \cos y}{\sin y}
$$

and claim the following:

1. For $0<\tau<\pi, F_{\tau}(y)$ decreases for $y \in(\tau, \pi)$ from $\infty$ to $-\infty$.

2. For $\tau=0, F_{\tau}(y)$ decreases for $y \in(\tau, \pi)$ from 1 to $-\infty$.

3. For $\tau<0, F_{\tau}(y)$ decreases for $y \in(0, \pi)$ from $\infty$ to $-\infty$.

It is easy to see that $\lim _{y \rightarrow \pi_{-}} F_{\tau}(y)=-\infty, \lim _{y \rightarrow \tau_{+}} F_{\tau}(y)=\infty$ for $\tau>0$ and $\lim _{y \rightarrow 0_{+}} F_{0}(y)=1$. Furthermore, $\lim _{y \rightarrow 0_{+}} F_{\tau}(y)=\infty$ for $\tau<0$ since the first term in $F_{\tau}(y)$ has a logarithmic singularity. To verify $1 ., 2$. and 3 . we need to show that $F_{\tau}^{\prime}(y)<0$. We find, after some computation,

$$
F_{\tau}^{\prime}(y)=\frac{1}{\sin y}\left(2 \cos y-\frac{\sin y}{y-\tau}-\frac{y-\tau}{\sin y}\right) .
$$

Putting $\kappa=(\sin y) /(y-\tau)$ then $\kappa>0$ and hence $\kappa+1 / \kappa \geq 2$. Since $\cos y<1$ it follows that indeed $F_{\tau}^{\prime}(y)<0$.

From 1. and 3. it follows that for given $\sigma \in \mathbb{R}$ and $\tau<\pi, \tau \neq 0$ there is a unique solution $y$ to the equation $F_{\tau}(y)=\sigma$ and therefore there is a unique solution $x+i y \in \mathcal{S}_{2 \pi}$ to $f(x+i y)=\sigma+i \tau$. If $\tau=0$ there is by 2 . a unique solution $y$ to the equation $F_{\tau}(y)=\sigma$ when $\sigma<1$ and none when $\sigma \geq 1$.

For $\pi<y<2 \pi$ we have $\sin y<0$ so $\tau>y$ and therefore

$$
f(\{\pi<\Im z<2 \pi\}) \subseteq\{\pi<\Im w\} .
$$

For $\tau>\pi$ we put

$$
F_{\tau}(y)=\log \frac{-\sin y}{\tau-y}+\frac{(\tau-y) \cos y}{-\sin y} .
$$

It follows that $F_{\tau}(y)$ increases with $y \in(\pi, \min (\tau, 2 \pi))$, and the conformality follows in the same way as for the case $\tau<\pi$. $\square$

In the following we let $f^{-1}$ denote the inverse of the conformal mapping $f$ of Lemma 5.2, including its obvious continuous extension to both sides of the cuts.

LEMma 5.3. The function $w \mapsto f^{-1}(1-w)$ is a Pick function with the representation

$$
f^{-1}(1-w)=\Re f^{-1}(1-i)+\int_{0}^{\infty}\left(\frac{1}{t-w}-\frac{t}{t^{2}+1}\right) \eta(t) d t
$$

where

$$
\eta(t)=\frac{1}{\pi} \Im f^{-1}(1-t)
$$

is increasing on $(0, \infty)$ from 0 to 1 . 
Proof. First of all, $1-w$ belongs to the lower half plane when $w$ belongs to the upper half plane. Since $f^{-1}$ maps all of $\mathcal{T}$, and hence in particular the lower half plane, into the strip $0<\Im z<2 \pi, f^{-1}(1-w)$ is certainly a Pick function. We next derive its integral representation. Since its imaginary part is bounded the number $a$ in the representation (16) must be zero.

It remains to identify the measure $\mu$. Since the function $f^{-1}(1-t-i y)$ is continuous for $(t, y) \in \mathbb{R} \times[0,1]$ and is real for $y=0$ and $t<0$ we find

$$
\eta(t)=\lim _{y \rightarrow 0_{+}} \frac{\Im f^{-1}(1-t-i y)}{\pi}=\frac{\Im f^{-1}(1-t)}{\pi}
$$

for $t>0$ and $\eta(t)=0$ for $t<0$.

By definition the function $Y(t)=\pi \eta(t)$ satisfies the equation

$$
F_{0}(Y(t))=\log \frac{\sin Y(t)}{Y(t)}+\frac{Y(t) \cos Y(t)}{\sin Y(t)}=1-t
$$

(with the notation of Lemma 5.2) and therefore

$$
Y^{\prime}(t) F_{0}^{\prime}(Y(t))=-1 \text {. }
$$

Since $F_{0}^{\prime}$ is negative (see again Lemma 5.2), $Y(t)$ and hence $\eta(t)$ must be increasing. Since $Y(t)$ tends to $\pi$ as $t$ tends to $\infty$ (this is because $F_{0}(Y(t)) \rightarrow-\infty$ ), $\eta(t) \rightarrow 1$ as $t \rightarrow \infty$. $\mathrm{B}$

Lemma 5.4. The function

$$
g(w)=f^{-1}(w)+\overline{f^{-1}(\bar{w})}
$$

has a holomorphic extension to $\mathbb{C} \backslash\{\Im w= \pm 2 \pi$, $\mathrm{sw} \geq 1\}$. All points on the lines $\{\Im w= \pm 2 \pi, \Re w \geq 1\}$ are singular points for $g$.

Proof. Since $f^{-1}$ is a conformal mapping of the region

$$
\mathbb{C} \backslash(\{\Im w=2 \pi, \Re w \geq 1\} \cup\{\Im w=0, \Re w \geq 1\}),
$$

the function $g$ is holomorphic in

$$
\mathbb{C} \backslash(\{\Im w= \pm 2 \pi, \Re w \geq 1\} \cup\{\Im w=0, \Re w \geq 1\}) .
$$

However, $g$ has a continuous extension to $\{\Im w=0, \Re w \geq 1\}$ with boundary values $u+v$ (see the beginning of Section 3), so from Moreras theorem we conclude that $g$ is holomorphic across this half-line. Concerning the boundary values on the half-lines $\{\Im w= \pm 2 \pi, \Re w \geq 1\}$ we have $(\sigma \geq 1)$

$$
\begin{aligned}
\lim _{\tau \rightarrow 2 \pi_{-}} g(\sigma+i \tau) & =\lim _{\tau \rightarrow 2 \pi_{-}} f^{-1}(\sigma+i \tau)+\overline{f^{-1}(\sigma-2 \pi i)} \\
& =v(\sigma)+2 \pi i+\overline{f^{-1}(\sigma-2 \pi i)}
\end{aligned}
$$

and similarly

$$
\lim _{\tau \rightarrow 2 \pi_{+}} g(\sigma+i \tau)=u(\sigma)+2 \pi i+\overline{f^{-1}(\sigma-2 \pi i)} .
$$

Therefore $g$ is not continuous across any segment of the half-line

$$
\{\Im w=2 \pi, \Re w \geq 1\}
$$


and so all these points are singular points. Since $g(w)=\overline{g(\bar{w})}$ the same conclusion holds for the points on the other half-line.

The next result relates $g$ from the lemma above with $u, v$ (defined by (7)) and $\xi$.

COROllary 5.5. The function

$$
g(w)=f^{-1}(w)+\overline{f^{-1}(\bar{w})}
$$

is a holomorphic extension of $u+v$ to $\mathbb{C} \backslash\{\Im w= \pm 2 \pi, \Re w \geq 1\}$. The function $g^{\prime}$ is thus a holomorphic extension of $\xi$.

Proposition 5.6. The Taylor series for $\xi(z)$ centered at 1 has radius of convergence equal to $2 \pi$ and the asymptotic series

$$
\sum_{k=0}^{\infty} \frac{\xi^{(k)}(1)}{z^{k}}
$$

diverges for any $z$ in $\mathbb{C}$.

Proof. We have $\xi=g^{\prime}$, where $g$ is the function in Lemma 5.4. If the radius of convergence of $\xi$ at 1 were larger than $2 \pi$ then the primitive $g$ would also have a holomorhic extension to this larger disk, and this contradicts the fact that $g$ has singular points in that disk.

Concerning the divergence of the asymptotic series we use that the radius of convergence of the Taylor series is finite. It means that

$$
\limsup _{k \rightarrow \infty}\left(\frac{\left|\xi^{(k)}(1)\right|}{k !}\right)^{1 / k}>0,
$$

and hence that for some $\epsilon>0,\left|\xi^{(k)}(1)\right|^{1 / k} /(k !)^{1 / k}>\epsilon$ for infinitely many $k$. Stirlings formula yields $(k !)^{1 / k} \sim k / e$ and therefore

$$
\limsup _{k \rightarrow \infty}\left|\xi^{(k)}(1)\right|^{1 / k}=\infty .
$$

This shows on the other hand that the asymptotic series diverges for any complex number $z$. $\mathrm{c}$

In the following $\log$ denotes the principal logarithm defined in the cut plane $\mathbb{C} \backslash(-\infty, 0]$.

Lemma 5.7. The function

$$
\Psi(w)=f^{-1}(\log w)+\overline{f^{-1}(\log \bar{w})}
$$

is a Pick function. It has the representation

$$
\Psi(w)=\Re \Psi(i)-\int_{0}^{\infty}\left(\frac{1}{t+w}-\frac{t}{t^{2}+1}\right) h(t) d t,
$$

where the function $h$ is given as

$$
h(t)=1-\frac{\Im f^{-1}(\log t-i \pi)}{\pi} .
$$


Furthermore, $h$ increases on $(0, \infty)$ from 0 to 1.

Proof. We consider the holomorphic function $g$ from Lemma 5.4 in the strip $\mathcal{S}=\{0<\Im w<\pi\}$. Hence the function $V(w)=\Im g(w)$ is a harmonic function in $\mathcal{S}$. Furthermore, since $f^{-1}$ maps all of $\{\Im w<\pi\} \backslash\{x \mid x \geq 1\}$ into $\mathcal{S}, V$ is also bounded there, with the apriori bound $-\pi<V(w)<\pi$. We claim that indeed $0<V(w)<\pi$ for all $w \in \mathcal{S}$.

We consider the boundary values of $V$. The horizontal line $\{t+i \pi, \mid t \in \mathbb{R}\}$ is mapped by $f^{-1}$ to itself, whereas $\{t-i \pi, \mid t \in \mathbb{R}\}$ is mapped by $f^{-1}$ to some curve inside the strip $\mathcal{S}$. Therefore $\overline{f^{-1}(t-i \pi)}$ has imaginary part greater than $-\pi$ and hence the boundary values $V(t+i \pi), t \in \mathbb{R}$, are all non negative. Since $g(\bar{w})=\overline{g(w)}$, the boundary values $V(t), t \in \mathbb{R}$, are all zero. Therefore $V$ has non-negative boundary values. Since it is bounded, the maximum principle in an unbounded region (see e.g. [12]) yields that $V(w)>0$ for all $w \in \mathcal{S}$.

Since log maps the upper half plane onto the strip $\mathcal{S}$, the function $\Psi(w)=g(\log w)$ maps the upper half plane into itself, and is hence a Pick function. Since $\Im \Psi$ is bounded, $\Psi$ has an integral representation of the form

$$
\Psi(w)=\Re \Psi(i)+\int_{-\infty}^{\infty}\left(\frac{1}{t-w}-\frac{t}{t^{2}+1}\right) d \mu(t),
$$

for some positive measure $\mu$. Here $\Im \Psi(t)=0$ for $t>0$ since $V$ is zero on the real line. We thus find the measure $\mu$ to be supported on the negative half-line with density

$$
1-\frac{\Im f^{-1}(\log (-t)-i \pi)}{\pi}, \quad t<0 .
$$

After making a change of variable $(t \mapsto-t)$ in the integral it follows that

$$
\Psi(w)=\Re \Psi(i)-\int_{0}^{\infty}\left(\frac{1}{t+w}-\frac{t}{t^{2}+1}\right) h(t) d t
$$

where $h$ is the function in the statement of the lemma.

By definition of $h, \pi(1-h(t))=\Im f^{-1}(\log t-i \pi)$, so $h$ is increasing if the solution $Y(t)$ to the equation $F_{-\pi}(Y(t))=\log t$ is decreasing. This is indeed the case, since

$$
F_{-\pi}^{\prime}(Y(t)) Y^{\prime}(t)=\frac{1}{t}>0
$$

and $F_{-\pi}^{\prime}<0$.

Proposition 5.8. The function $\xi(\log w)$ is holomorphic in the cut plane $\mathbb{C} \backslash$ $(-\infty, 0]$ with the representation

$$
\xi(\log w)=1-\int_{0}^{\infty} \frac{t}{t+w} h^{\prime}(t) d t
$$

where $h(t)$ is given in Lemma 5.\%.

Proof. From Corollary 5.5 it follows that $\Psi(w)=u(\log w)+v(\log w)$, for $w \geq e$, where $\Psi$ is the function in Lemma 5.7. Hence $\xi(\log w)=w \Psi^{\prime}(w)$ and so we get from (19),

$$
\xi(\log w)=\int_{0}^{\infty} \frac{w h(t)}{(t+w)^{2}} d t
$$


Integration by parts on the right-hand side of this relation yields

$$
\xi(\log w)=\int_{0}^{\infty} \frac{w}{t+w} h^{\prime}(t) d t
$$

or

$$
\xi(\log w)=1-\int_{0}^{\infty} \frac{t}{t+w} h^{\prime}(t) d t .
$$

Proof of Proposition 3.2.

$\xi(t)$ increases from 0 to 1 for $t \in \mathbb{R}$ (this is more than is stated in the proposition). From (8), $\lim _{t \rightarrow \infty} \xi(t)=1$. Letting $w \rightarrow 0_{+}$in the integral in Proposition 5.8 then

$$
\xi(\log w) \rightarrow 1-\int_{0}^{\infty} h^{\prime}(t) d t=1-\lim _{t \rightarrow \infty} h(t)+h(0)=0 .
$$

Hence $\xi(t) \rightarrow 0$ as $t \rightarrow-\infty$. Differentiation of the formula in Proposition 5.8 gives us

$$
\frac{\xi^{\prime}(\log w)}{w}=\int_{0}^{\infty} \frac{t}{(t+w)^{2}} h^{\prime}(t) d t
$$

and since $h^{\prime}(t)>0$ for all $t \in \mathbb{R}$, we see that $\xi^{\prime}(t)>0$ for all $t \in \mathbb{R}$.

$\xi(t)$ is concave for $t \geq 1$. Differentiation of both sides of the relation above yields

$$
\xi^{\prime \prime}(\log w)-\xi^{\prime}(\log w)=-2 w^{2} \int_{0}^{\infty} \frac{t}{(t+w)^{3}} h^{\prime}(t) d t,
$$

so that, again using the integral representation of $\xi^{\prime}(\log w)$,

$$
\begin{aligned}
\xi^{\prime \prime}(\log w) & =\int_{0}^{\infty}\left(\frac{-2 w^{2}}{(t+w)^{3}}+\frac{w}{(t+w)^{2}}\right) t h^{\prime}(t) d t \\
& =\int_{0}^{\infty} \frac{t-w}{(t+w)^{3}} w t h^{\prime}(t) d t \\
& =w \int_{0}^{\infty} \frac{s-1}{(s+1)^{3}} s^{\prime}(s w) d s .
\end{aligned}
$$

This last integral we split into two, one for $s<1$ and another for $s>1$. We thus get, after making the substitution $s \rightarrow 1 / s$ in the latter one,

$$
\frac{\xi^{\prime \prime}(\log w)}{w}=\int_{0}^{1} \frac{1-s}{(1+s)^{3}}\left(\frac{1}{s} h^{\prime}\left(\frac{w}{s}\right)-s h^{\prime}(s w)\right) d s .
$$

From this relation we see that $\xi$ is concave on $\left[\log w_{0}, \infty\right)$ if

$$
\frac{1}{s} h^{\prime}\left(\frac{w}{s}\right)-s h^{\prime}(s w) \leq 0 \quad \text { for } \quad s \in(0,1) \quad \text { and } \quad w \geq w_{0} .
$$

Now, as noted in the proof of Lemma $5.7, h(s)=1-Y_{-\pi}(\log s) / \pi$, where $Y_{-\pi}(t) \in$ $(0, \pi)$ is the solution to the equation $F_{-\pi}\left(Y_{-\pi}(t)\right)=t$, see (18). Therefore $h^{\prime}(s)=$ $-Y_{-\pi}^{\prime}(\log s) /(\pi s)$ and hence

$$
\frac{1}{s} h^{\prime}\left(\frac{w}{s}\right)=-\frac{Y_{-\pi}^{\prime}(\log w-\log s)}{\pi w}
$$


and

$$
s^{\prime}(s w)=-\frac{Y_{-\pi}^{\prime}(\log w+\log s)}{\pi w} .
$$

The relation (20) holds provided

$$
Y_{-\pi}^{\prime}(\log w-\log s) \geq Y_{-\pi}^{\prime}(\log w+\log s) .
$$

Since $\log s$ runs through $(-\infty, 0)$ when $s \in(0,1)$, this condition is the same as

$$
Y_{-\pi}^{\prime}(\log w+t) \geq Y_{-\pi}^{\prime}(\log w-t)
$$

for all $t \geq 0$ and all $w \geq w_{0}$. We aim at proving this for $w_{0}=e$, so what we really should prove is the following:

$$
Y_{-\pi}^{\prime}(\alpha+t) \geq Y_{-\pi}^{\prime}(\alpha-t)
$$

for all $t \geq 0$ and all $\alpha \geq 1$. This inequality is verified by using Lemma 5.3. According to that lemma, with $w=1-\alpha-t+i \pi$, we have

$$
\begin{aligned}
Y_{-\pi}(\alpha+t) & \left.=\Im f^{-1}(\alpha+t-i \pi)\right) \\
& =\Im f^{-1}(1-(1-\alpha-t+i \pi)) \\
& =\int_{0}^{\infty} \frac{\pi}{(s+t+\alpha-1)^{2}+\pi^{2}} \eta(s) d s
\end{aligned}
$$

so that

$$
\begin{aligned}
Y_{-\pi}^{\prime}(\alpha+t)= & \int_{0}^{\infty}\left(\frac{\partial}{\partial t} \frac{\pi}{(s+t+\alpha-1)^{2}+\pi^{2}}\right) \eta(s) d s \\
= & \int_{0}^{\infty}\left(\frac{\partial}{\partial s} \frac{\pi}{(s+t+\alpha-1)^{2}+\pi^{2}}\right) \eta(s) d s \\
= & {\left[\frac{\pi}{(s+t+\alpha-1)^{2}+\pi^{2}} \eta(s)\right]_{0}^{\infty} } \\
& -\int_{0}^{\infty} \frac{\pi}{(s+t+\alpha-1)^{2}+\pi^{2}} \eta^{\prime}(s) d s \\
= & -\int_{0}^{\infty} \frac{\pi}{(s+t+\alpha-1)^{2}+\pi^{2}} \eta^{\prime}(s) d s .
\end{aligned}
$$

Replacing $t$ by $-t$ in this formula we get

$$
Y_{-\pi}^{\prime}(\alpha-t)=-\int_{0}^{\infty} \frac{\pi}{(s-t+\alpha-1)^{2}+\pi^{2}} \eta^{\prime}(s) d s
$$

so that

$$
\begin{aligned}
& Y_{-\pi}^{\prime}(\alpha+t)-Y_{-\pi}^{\prime}(\alpha-t) \\
& =4 \pi t \int_{0}^{\infty} \frac{s+\alpha-1}{\left((s+t+\alpha-1)^{2}+\pi^{2}\right)\left((s-t+\alpha-1)^{2}+\pi^{2}\right)} \eta^{\prime}(s) d s .
\end{aligned}
$$

Since $\eta^{\prime}(s)>0$ it follows that $Y_{-\pi}^{\prime}(\alpha+t)-Y_{-\pi}^{\prime}(\alpha-t)$ has the same sign as $t$ for $\alpha \geq 1$ and hence is positive for $t>0$. 
Behaviour of $\xi$ at $\infty$. The behaviour of $\xi$ and its derivatives at infinity can be investigated through the theory of completely monotonic functions. The investigation is based on a simple lemma of independent interest.

We recall that a $C^{\infty}$-function $\nu(t)$ defined on the positive half-line is called completely monotonic if

$$
(-1)^{p} \nu^{(p)}(t) \geq 0, \quad \text { for all integers } \quad p \geq 0 .
$$

It is called a Bernstein function if

$$
\nu(t) \geq 0 \quad \text { and } \quad(-1)^{p} \nu^{(p)}(t) \leq 0, \quad \text { for all integers } \quad p \geq 1 .
$$

The last conditions can also be expressed that $\nu^{\prime}(t)$ is completely monotonic. For details about these classes of functions see e.g. [5].

Lemma 5.9. Let $F$ be completely monotonic, let $\varsigma(t)$ be a positive $C^{\infty}$-function for $t>0$ and assume that $\varsigma^{\prime}(t)=F(\varsigma(t))$. Then:

For each $n \geq 1$ there exists a completely monotonic function $F_{n}$ such that

$$
(-1)^{n-1} \varsigma^{(n)}(t)=F_{n}(\varsigma(t)) .
$$

In particular $\varsigma$ is a Bernstein function.

Proof. For $n=1, F_{1}=F$ can be used. If $(-1)^{n-1} \varsigma^{(n)}(t)=F_{n}(\varsigma(t))$ for some completely monotonic function $F_{n}$ then

$$
(-1)^{n} \varsigma^{(n+1)}(t)=-F_{n}^{\prime}(\varsigma(t)) \varsigma^{\prime}(t)=-F_{n}^{\prime}(\varsigma(t)) F(\varsigma(t)) .
$$

Here $F_{n+1} \equiv-F_{n}^{\prime} F$ is completely monotonic as a product of two completely monotonic fuctions.

Furthermore we have

$$
(-1)^{n} \varsigma^{(n)}(t)=-F_{n}(\varsigma(t)) \leq 0,
$$

so $\varsigma$ is a Bernstein function.

Proposition 5.10. The functions $-u(t+1)$ and $v(t+1)$ are Bernstein functions and

$$
\lim _{t \rightarrow \infty} v(t) / t=1, \quad \lim _{t \rightarrow \infty} u(t) / t=0 .
$$

Proof. We have $v^{\prime}(t)=F(v(t))$ where

$$
F(x)=\frac{1}{1-e^{-x}}=\sum_{k=0}^{\infty} e^{-k x}
$$

is completely monotonic. Since $v(t) \rightarrow \infty$ as $t \rightarrow \infty$ we have $e^{-v(t)} \rightarrow 0$ and hence $v(t) / t=1-e^{-v(t)} / t \rightarrow 1$ as $t \rightarrow \infty$.

If $w \equiv-u$ then $w^{\prime}(t)=G(w(t))$ where

$$
G(x)=\frac{1}{e^{x}-1}=\sum_{k=1}^{\infty} e^{-k x}
$$


is completely monotonic. Furthermore, $t=e^{-u(t)}+u(t)>1+u(t)^{2} / 2($ since $u(t)<0)$ so that $-u(t)<\sqrt{2(t-1)}$ and thus $u(t) / t \rightarrow 0$ as $t \rightarrow \infty$.

Bernstein functions admit integral representations (see e.g. [5, p. 64]) and thus we have

$$
v(t+1)=t+\int_{0}^{\infty}\left(1-e^{-x t}\right) d \lambda(x)
$$

and

$$
-u(t+1)=\int_{0}^{\infty}\left(1-e^{-x t}\right) d \sigma(x)
$$

for some positive measures $\lambda$ and $\sigma$ on $(0, \infty)$. Since $t+1-v(t+1)=e^{-v(t+1)} \rightarrow 0$ for $t \rightarrow \infty$ we conclude that $\lambda((0, \infty))=1$ and hence

$$
v(t+1)=t+1-\int_{0}^{\infty} e^{-x t} d \lambda(x)
$$

The measure $\sigma$ has infinite total mass because $u(t) \rightarrow-\infty$ for $t \rightarrow \infty$.

From these representations we rediscover the relation $\xi(t)\left(=u^{\prime}(t)+v^{\prime}(t)\right) \rightarrow 1$ as $t \rightarrow \infty$ (see (8)). Furthermore, $u^{(n+1)}(t)$ and $v^{(n+1)}(t)$ both tend to zero as $t$ tends to infinity for any $n \geq 1$. Consequently, $\xi^{(n)}(t) \rightarrow 0$ as $t \rightarrow \infty$ (for any $n \geq 1$ ) and Lemma 4.4 is proved.

One could hope to deduce the monotonicity properties of $\xi$ by using these integral representations. We have not succeeded in doing this, since much cancellation between $u$ and $v$ takes place and we do not know $\lambda$ and $\sigma$ explicitly. Furthermore, it is not even true that $\xi(t+1)$ is a Bernstein function even though it is increasing and concave.

6. Appendix: Higher order expansions. In this section the asymptotic expansion of $m(x)$ up to $o\left(x^{-9}\right)$ can be found. We have also included the expansion of the auxiliary function $\varphi(x)$ and the numbers $\xi^{(k)}(1)$ for $k$ up to 10. These results were found using the Maple 9 program. The Maple code and a short description can be found at

$$
\text { www . math. ku.dk/ berg/ }
$$

The asymptotic expansion of $m(x)$ is

$$
m(x)=x-\frac{1}{3}+\sum_{k=1}^{9} \frac{m_{k}}{x^{k}}+o\left(x^{-9}\right)
$$


where

$$
\begin{aligned}
& m_{1}=\frac{2^{3}}{3^{4} \cdot 5} \\
& m_{2}=\frac{2^{3} \cdot 23}{3^{6} \cdot 5 \cdot 7} \\
& m_{3}=\frac{2^{3} \cdot 281}{3^{9} \cdot 5^{2} \cdot 7} \\
& m_{4}=-\frac{2^{3} \cdot 17 \cdot 139753}{3^{13} \cdot 5^{3} \cdot 7 \cdot 11} \\
& m_{5}=-\frac{2^{3} \cdot 708494947}{3^{15} \cdot 5^{3} \cdot 7^{2} \cdot 11 \cdot 13} \\
& m_{6}=\frac{2^{3} \cdot 140814348739}{3^{18} \cdot 5^{4} \cdot 7^{2} \cdot 11 \cdot 13} \\
& m_{7}=\frac{2^{3} \cdot 7663181003289047}{3^{21} \cdot 5^{6} \cdot 7^{3} \cdot 11 \cdot 13 \cdot 17} \\
& m_{8}=-\frac{2^{3} \cdot 653 \cdot 1359581 \cdot 759929 \cdot 3307}{3^{23} \cdot 5^{6} \cdot 7^{3} \cdot 11 \cdot 13 \cdot 17 \cdot 19} \\
& m_{9}=-\frac{2^{3} \cdot 29 \cdot 1376560394479059407}{3^{27} \cdot 5^{7} \cdot 7^{3} \cdot 11^{2} \cdot 17} .
\end{aligned}
$$

The asymptotic expansion of $\varphi(x)$ is

$$
\varphi(x)=\sum_{k=1}^{10} \frac{c_{k}}{x^{k}}+o\left(x^{-10}\right),
$$

where

$$
\begin{aligned}
& c_{1}=\frac{1}{3} \\
& c_{2}=\frac{29}{3^{4} \cdot 5 \cdot 2} \\
& c_{3}=-\frac{37}{3^{6} \cdot 5 \cdot 7} \\
& c_{4}=-\frac{3877}{3^{9} \cdot 5^{2} \cdot 2^{2}} \\
& c_{5}=\frac{8957413}{3^{13} \cdot 5^{3} \cdot 7 \cdot 11} \\
& c_{6}=\frac{401 \cdot 8842279}{2 \cdot 3^{15} \cdot 5^{2} \cdot 7^{2} \cdot 11 \cdot 13} \\
& c_{7}=-\frac{356146891 \cdot 2039}{3^{18} \cdot 5^{4} \cdot 7^{2} \cdot 11 \cdot 13} \\
& c_{8}=-\frac{216607304027 \cdot 3077479}{2^{3} \cdot 3^{21} \cdot 5^{6} \cdot 7^{3} \cdot 11 \cdot 13 \cdot 17} \\
& c_{9}=\frac{31 \cdot 743 \cdot 4569027042343}{3^{23} \cdot 5^{3} \cdot 7^{3} \cdot 11 \cdot 13 \cdot 17 \cdot 19} \\
& c_{10}=\frac{71 \cdot 282699240672481 \cdot 1949 \cdot 5113}{2 \cdot 3^{27} \cdot 5^{7} \cdot 7^{3} \cdot 11^{2} \cdot 13 \cdot 17 \cdot 19} .
\end{aligned}
$$


The derivatives of $\xi$ are:

$$
\begin{aligned}
\xi(1) & =\frac{2}{3} \\
\xi^{\prime}(1) & =\frac{2^{3}}{3^{3} \cdot 5} \\
\xi^{(2)}(1) & =-\frac{2^{4}}{3^{4} \cdot 5 \cdot 7} \\
\xi^{(3)}(1) & =-\frac{2^{5}}{3^{5} \cdot 5 \cdot 7} \\
\xi^{(4)}(1) & =\frac{2^{6} \cdot 281}{3^{8} \cdot 5^{2} \cdot 7 \cdot 11} \\
\xi^{(5)}(1) & =\frac{2^{7} \cdot 23 \cdot 227}{3^{9} \cdot 5^{2} \cdot 7 \cdot 11 \cdot 13} \\
\xi^{(6)}(1) & =-\frac{2^{8} \cdot 53 \cdot 103}{3^{10} \cdot 5^{2} \cdot 7 \cdot 11 \cdot 13} \\
\xi^{(7)}(1) & =-\frac{2^{9} \cdot 373 \cdot 4439 \cdot 557}{3^{12} \cdot 5^{4} \cdot 7^{2} \cdot 11 \cdot 13 \cdot 17} \\
\xi^{(8)}(1) & =\frac{2^{10} \cdot 2650986803}{3^{13} \cdot 5^{4} \cdot 7^{2} \cdot 11 \cdot 13 \cdot 17 \cdot 19} \\
\xi^{(9)}(1) & =\frac{2^{11} \cdot 6171801683}{3^{14} \cdot 5^{4} \cdot 7^{2} \cdot 11 \cdot 13 \cdot 17 \cdot 19} \\
\xi^{(10)}(1) & =-\frac{2^{12} \cdot 1117 \cdot 3835213201}{3^{16} \cdot 5^{2} \cdot 7^{2} \cdot 11 \cdot 13 \cdot 17 \cdot 19 \cdot 23} .
\end{aligned}
$$

Added in Proof. The conjecture $m^{\prime \prime}(x)>0$ mentioned at the end of Section 1 has been proved by the authors.

\section{REFERENCES}

[1] J. A. Adell And P. JodrÁ, Sharp estimates for the median of the $\Gamma(n+1,1)$ distribution, Statistics and Probability Letters, 71 (2005), pp. 185-191.

[2] S. E. Alm, Monotonicity of the difference between median and mean of gamma distributions and of a related Ramanujan sequence, Bernoulli, 9:2 (2003), pp. 351-371.

[3] H. Alzer, Proof of the Chen-Rubin conjecture, Proc. Roy. Soc. Edinburgh Sect. A, 135:4 (2005), pp. $677-688$.

[4] G. E. Andrews, R. Askey, R. Roy, Special functions, Cambridge University Press, Cambridge, 1999.

[5] C. Berg and G. Forst, Potential Theory on Locally Compact Abelian Groups, Springer, Berlin-Heidelberg-New York, 1975.

[6] Bruce C. Berndt, Youn-Seo Chol And Soon-Yi Kang, The problems submitted by Ramanujan to the journal of the Indian Mathematical society. In: Continued Fractions: From analytic number theory to constructive approximation (Columbia, MO, 1998), Contemporary Mathematics, 236, Amer. Math. Soc., Providence R.I. (1999), pp. 15-56.

[7] J. Chen and H. Rubin, Bounds for the difference between median and mean og Gamma and Poisson distributions, Statist. Probab. Lett., 4 (1986), pp. 281-283.

[8] K. P. CHOI, On the medians of gamma distributions and an equation of Ramanujan, Proc. AMS, 121 (1994), pp. 245-251.

[9] W. F. Donoghue, JR., Monotone matrix functions and analytic continuation, Springer, BerlinHeidelberg-New York, 1974.

[10] P. Flajolet, P. J Grabner, P. Kirschenhofer, H. Prodinger, On Ramanujan's Q-function, J. Comput. Appl. Math., 58 (1995), pp. 103-116.

[11] J. Marsaglia, C249: The incomplete gamma function and Ramanujan's rational approximation to $e^{x}$. In: Comments, Conjectures and Conclusions, eds. I. Good, J. Statist. Comput. Simul., 24 (1986), pp. 163-169.

[12] P. Koosis, The Logarithmic Integral I, Cambridge University Press, Cambridge, 1988. 
[13] S. Ramanujan, Collected Papers, Question 294, Cambridge 1927.

[14] F. W. Steutel, K. van Harn, Infinite divisibility of probability distributions on the real line, Monographs and Textbooks in Pure and Applied Mathematics 259, Marcel Dekker, Inc., New York 2004.

[15] G. SzEGÖ, Über einige von S. Ramanujan gestellte Aufgaben, J. London Math. Soc., 3 (1928), pp. $225-232$.

[16] G. N. Watson, Theorems stated by Ramanujan (V): Approximations connected with $e^{x}$, Proc. London Math. Soc. (2), 29 (1929), pp. 293-308. 